\title{
THE “PLACEBO / NOCEBO EFFECT”: LEARNING THE IMPACT OF POSITIVE / NEGATIVE EMOTION (S) ON THE PHYSIOLOGY OF HUMAN CELLS
}

\author{
VIJAY A. KANADE
}

Research Associate, Evalueserve SEZ (Gurgaon) Private Limited, Haryana, India

\begin{abstract}
Placebo effect is an epidemic phenomenon observed in subjects who perceive an improvement in his/her medical condition due to personal beliefs, rather than the drug treatment itself. Placebos play a significant role in medical research. Common placebos administered by the doctors include inert tablets (like sugar pills), shame surgery (placebo surgery), and other procedures opted by providing false information. Placebo effects are prominently driving the scientific research aiming to unravel the underlying neurobiological facets of action in pain relief, Parkinson's disease, depression etc. Placebos have been observed to impact the physiological changes in human body, such as heart rate, blood pressure accompanied by the chemical activity in the brain, in medical cases which involve pain, anxiety, fatigue, etc. The placebo effect projects the notable influence of perception and brain's role in the physical health of a human. However, the question of how placebos work has still not been answered with utmost surety by medical science. The paper proposes a unified platform for studying, factoring and visualizing the underlying effects of placebo/nocebo on human cell physiology, wherein the nocebo effect is the negative version of placebo effect.

KEYWORDS: Placebo / Nocebo effect, Brain Computer Interface (BCI), Electroencephalogram (EEG), Biodegradable Silicon Membrane Implant \& On-Skin Electrode
\end{abstract}

Received: Oct 14, 2017; Accepted: Nov 06, 2017; Published: Dec 06, 2017; Paper Id.: IJMPSDEC20174

\section{INTRODUCTION}

The 'Placebo / Nocebo effect' is a term used to outline the episode when a patient's symptoms are mitigated by an ineffectual treatment, solely because the individual believes that the medication will work. This very aspect of human psychology exhibits the power of the human mind. Allowing one's self to recover just on the basis of belief that something is working, makes you doubt the true effectiveness of the futile yet real medication used. Placebo effects surface not just from a conscious belief in the medicines but also from a subconscious connection between the thought of recovering and the experience of being treated [1]. Placebo effect is been observed in innumerable medical cases, yet the phenomenon remains to be a mystery for the medical fraternity as well as the scientists trying to decode the effects and reasons for its appearance.

Conventional medicine is still not zeroing-on the significant role energy plays as "information" in the complex biological network within a human body - however non-invasive scanning technologies, which read such energy fields, have been accepted gracefully. Quantum physics lead to the emergence of scanning devices that read and analyze the frequencies emitted by specific chemicals. These scanning methods pave the way for identifying the molecular composition of materials / molecules / objects. Physicists have customized these devices to comprehend the energy spectra emitted by our body's tissues / cells and organs. Research has revealed and verified the facts that diseased tissue emits its own distinctive and unique energy signature pattern, which differs from the 
energy emitted by surrounding wholesome and healthy cells. The energy signatures that pass through our bodies propagate through space as invisible energy waves that resemble ripples on a water surface. These energy fields are used by the modern devices (CAT scans, MRIs, etc.) to detect diseases non-invasively.

This paper illustrates a novel invention in the field of cognitive neuroscience that investigates the impacts (or effects) of positive and negative emotions (or thoughts i.e. Joyful, Angry, Protected, Sad, Surprised, Fear, Satisfied and Unconcerned) on human cells (i.e. cancerous, tumor cells, or any normal cell/tissue). The paper proposes a unique technique of tracking the energy fields / signatures emitted by human cells in order to localize and study the paramount effect of thoughts on body cells / tissues and organs.

\section{MEDICAL CASES}

Few of the interesting medical cases reveling the Placebo / Nocebo-effect are discussed below:

\section{Case 1: Placebo-Effect}

In 2002, a Baylor School of Medicine study published in the 'New England Journal of Medicine' an unusual case of patients suffering from severe knee pain, who had undergone an evaluated surgery (Moseley, et al, 2002) [6]. The author of the case study, Dr. Bruce Moseley, was pretty much sure that knee surgery would help his patients recover: All medical surgeons consider it obvious that there cannot practically be a placebo effect in a hard-core surgery case. But Dr. Moseley was trying to understand the actual sub-sections of the surgery giving relief to his patients. The patients involved in the study were divided into three subgroups. For each subgroup Moseley adopted a different technique. For subgroup one, Moseley shaved the damaged cartilage in the knee. For the second subgroup, he removed the material thought to be causing the inflammatory effect by flushing out the knee joint. For the third subgroup, he chose a shame or "fake" surgery. The patients under this subgroup were sedated, followed by marking three standard incisions and then conversed just as like they would have gone under the same drill during a real surgery - Dr. Moseley further splashed salt water to activate the sound of knee-washing powder. After 40 minutes (approx.), the incisions were sewed up as if the patients had undergone the real surgery. The three subgroups were prescribed the same postoperative treatment \& medication, which covered an exercise program.

The results were freakish. Yes, the subgroups who received surgery, undoubtedly improved. However, the placebo subgroup improved in an equivalent proportion - as like the other two groups! In a period of time, the members of placebo subgroup were doing activities which they reported they could not do before the "surgery", like walking, playing basketball, etc. The placebo patients did not figure out whether they had gotten a real or a fake surgery, for a couple of years or so. Such is the effect of placebo. Studies have revealed the powerful impact of placebo in treating no. of diseases, including asthma, Parkinson's disease, depression, etc.

\section{Case 2: Nocebo-Effect}

If positive thinking can heal a damaged knee, or make a depressed person cheerful, consider the far reaching consequences of negative thinking. When the mind, through positive thinking shows positive signs on health, it is referred to as placebo effect. Conversely, when the same mind is involved in negative thought process, it can hamper the sound physical health. This effect is termed as nocebo effect.

In 2003, Discovery Health Channel broadcasted a program "Placebo: Mind over Medicine" [7]. One of the 
episodes showcased a Nashville physician, Clifton Meador, who had been contemplating \& observing the potential power of the nocebo effect for a considerable period of about 30 years. Dr. Meador had a patient in 1974, named Sam Londe, a retired shoe salesman suffering from esophageal cancer - a dreadful condition that was considered 100 percent lethal. On identification of the esophageal cancer, Londe was treated for it; however the entire medical team at that time knew that his esophageal cancer would not vanish with such ease and it would eventually recur. So no one was really surprised when Londe died a few weeks after his diagnosis drills.

However, the entire medical community was taken aback when an autopsy report was out and surprisingly showed that Londe had very little cancer in his body, which surely was not threatening enough to kill him. A couple of spots could be identified in the liver and one in the lung, but there was no evidence of the esophageal cancer that everyone presumed to have killed him.

What did Londe die of then? Had he died because he believed he was going to die? The case still haunts Meador three decades after Londe's death and remains a mystery to all.

\section{Case 3: The Language of Energy}

Energy waves have a behavioral pattern which is fundamental for biomedicine since the physical and chemical properties of an atom can be altered by using vibrational frequencies similar to physical signals like histamine and estrogen. Because it is the well known scientific fact that the atoms are in constant motion or vibration, they create certain wave patterns like the ones observed at a water lake - when the expanding ripples can be seen on dropping pebbles into the lake. Each atom has a unique vibration or frequency pattern due to the distribution of its negative and positive charges, coupled with its spin rate. (Oschman 2000) [8].

Scientists have identified a method to stop the vibration of an atom and conversely speed-up atom's vibrations by exploiting its energy waves. Initially the frequency of the target atom is identified and then the resulting destructive interference is utilized to cancel the atom's vibrations and stop it dead on its tracks i.e. from spinning. (Chu 2002; Rumbles 2001) [9], [10]. On similar lines, constructive interference is exploited for atoms to vibrate at speeds enough to break free from the bonds that glue them together. For example, the goblet atoms explode at a certain singing frequency of a vocalist.

Doctors channelize constructive interference for treating kidney stones, a rare case where the laws of quantum mechanics have been effectively used as a therapeutic tool in modern medicine. Kidney stones are crystal balls whose atoms jiggle at specific frequency. Doctors non-invasively focus a harmonic frequency on the crystal ball i.e. the kidney stone. Constructive interference occurs when the focused energy waves interact with the atoms in the kidney stones. Like the crystal in the goblet-vocalist example, the atoms of the kidney stones vibrate rapidly enough for the stones to explode and dissolve. The remaining smaller fragments can then be easily passed out from the system without any excruciating pain that accompanies larger stones.

On in-depth analysis of the above mentioned cases, it has been learnt that a standard method to quantify the effects of thoughts on the physical state of cells, tissues etc of a person isn't identified in modern science. Through this invention the effects of thoughts leading to a change in the way the body responds can be feasibly studied and analyzed.

\section{FRAMEWORK COMPONENTS}

The significant components for the entire framework of the invention include: 


\section{EEG Recording}

The method utilizes acoustic and visual stimuli to trigger the positive and negative emotions among the human test subjects. Further, the method uses Electroencephalogram (EEG) to extract the subject's brain signals from the conscious (i.e. targeting brain's topmost layers: neocortex and the lobes) and the subconscious mind (i.e. targeting limbic system and brain stem) for performing brainwave analysis. Thus, the invention encompasses the primary step of employing EEG-based emotion recognition by utilizing a wireless EEG headset.

EEG recording using International 10/20 system:

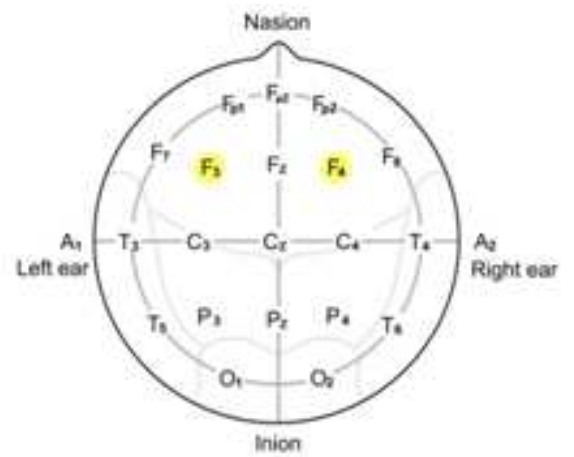

Figure 1: Focus Points in the Adopted Method Include F3 \& F4 From The Standard International 10/20 System [11].

\section{EMOTION IN THE BRAIN}

Stimuli enter the brain through the brain stem. The limbic system around the brain stem takes the responsibility for initial emotional interpretation of the entering signals from the nervous system. This sub-section of the brain further plays a significant part for motivation and memory functions.

The hypothalamus is a processing unit that processes the incoming signals and activates the corresponding visceral physiological effects, similar to a raised heart rate, blood pressure or galvanic skin response. Signals from the hypothalamus are then passed on to the amygdala, which is responsible for learning to connect stimuli to emotional reactions (reward / fear stratum) and for simulating a newer stimuli by matching them to past experience.

The amygdala is considered as a hub for emotion processing. However, the physical location of amygdala (i.e. like the limbic system) makes the EEG recordings awkward as the signals cannot be recorded or collected from the scalp of the patient. The amygdala possesses a connection to the temporal and prefrontal cortices, resulting in a consciously experienced feeling of an emotion.

The prefrontal lobe (directly behind the forehead) is involved in the so-called highest level of conscious thinking. It is held responsible for processing the cognitive, emotional and motivational signals. The prefrontal lobe is part of the frontal cortex, an emotional control center and that even determines personality traits. It is also involved in judgment and tracking of social behavior. So the prefrontal lobe is tracked by placing the electrodes in suitable position (F3 \& F4) for recording the brain signals.

The EEG recording is further processed to identify the emotional state of the patient in real-time. This includes standard BCI approach, wherein stimulus is given to the patient in the form of visual and audio sounds, EEG is recorded 
for that instance, the brain signals then undergo artifact filtering to remove unwanted noise signals, feature extraction is performed on the retrieved data and then the brain signals are classified for different emotional states of a patient.

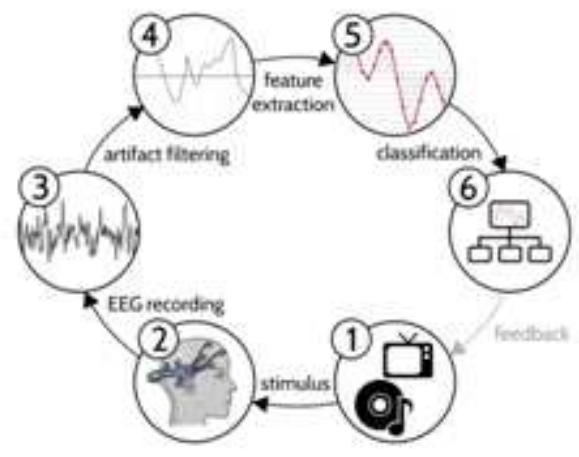

Figure 2: Standard BCI Approach

\section{Biodegradable Silicon Membrane Implant}

The method utilizes biodegradable silicon membrane implants carrying the miniature sensors. The biodegradable membrane is implanted at two specific locations: one membrane is implanted in the brain to track the changes in the brain ecosystem. The second membrane is implanted in the target affected area (i.e. area of tumor growth, area concerning cancerous cell growth, etc.) of the human body. A unique method is opted to embed the second membrane implant into the affected part. Initially the antibodies specific to the target molecules are attached to the surface of the membrane implant and the membrane is then implanted at a target location [5]. In the presence of target molecules, the implant binds to the target area and further causes the entire pack of target molecules (or cells) to clump together. This clumping event makes it easier to track / monitor the target cells of the affected body part.

The mentioned implants float around inside a human body for weeks after implantation - taking continuous uninterrupted measurements of swelling, intracranial pressure (within the skull), temperature, changes in chemical composition of body fluids, etc. around the target affected area. In addition, the implant holds the sensitive miniature antenna structure to note / determine the slightest of changes in the energy fields / vibration or frequency pattern emitted by the bodily tissues / cells on the immediate trigger of various emotions. This apparatus identifies the magnitude of the changing energy fields and communicates the values to the on-skin electrodes.

The silicon membrane implant possesses an inner layered segment to deliver medicines along with it to the target cells, so that the simultaneous effect of medicines and positive / negative thoughts on the cells can be tracked in real-time. All the while, the silicon sheets of the membrane get slowly dissolved by cerebrospinal fluid, body fluid surrounding the implant i.e. all of those juicy silicon atoms are reabsorbed by the body [2]. Since the 'silicon' ingredient isn't harmful to the human body, it liquefies and melts down to an ultimate zero-proportion. 


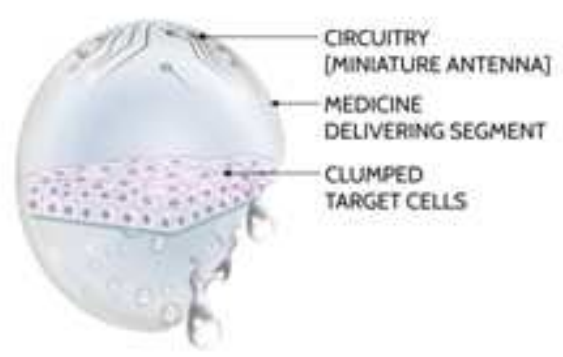

Figure 3: 3D Structure: Biodegradable Silicon Membrane Implant

The implant is placed in the target area of a patient which ought to be monitored - to facilitate the identification of impact of thoughts on bodily cells.

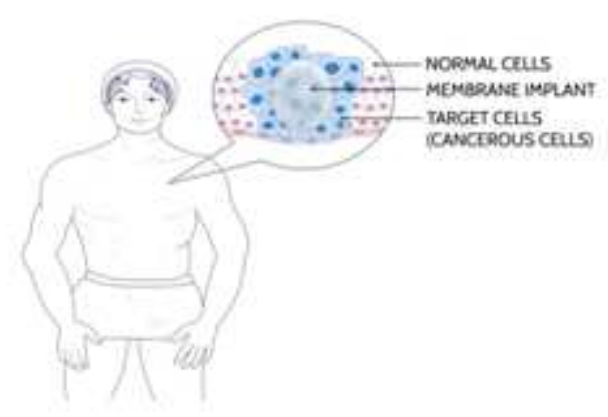

Figure 4: Configuring Implant Ecosystem

\section{On-Skin Electrodes}

The apparatus further consists of two on-skin electrodes positioned nearby to the implanted biodegradable silicon membrane. The implants mentioned above communicate with the on-skin electrodes by using the phenomenon of "Galvanic coupling". Thereby, the implants convey the information of its surrounding vicinity (i.e. temperature, pressure etc.) to the on-skin electrode periodically. Furthermore, these on-skin electrodes (i.e. electrode on the skull, as well as electrode close-by to target affected area like chest) are 'time-synched' with the EEG electrodes so as to unable the realtime visualization of cells (i.e. neurons as well as body cells, tissues or response seen through the change in the chemical composition of the body fluids, etc.) response to the positive / negative emotions tapped by the EEG device. Further, the electrode contains an antenna structure similar to the one in the implant for sensing the frequency pattern of affected (or diseased) body cells that continuously emit vibrational energies. This specific component is added to improve the accuracy of pinpointing the immediate change in the energy signature of the affected cells / tissues captured by the implant

Since the on-skin electrodes and the EEG electrodes are "time-synched", the current stature of a target cell / tissue along with the brains response can be studied / analyzed simultaneously at any particular instance of time. The on-skin electrode further transmits the collated 'time-synced' data wirelessly to the monitoring unit handled for a patient / subject.

\section{OPERATIVE MODEL}

The architecture utilized for understanding the above mentioned medical cases involve the 'Time-synced' operative model. To begin with, EEG signals of a patient are recorded by using the wireless EEG headset for the audio and visual stimuli provided to the patient. These EEG signals are processed and the emotion of the target subject is noted \& 
classified in real-time. While the emotion tracking procedure is 'on', the brain implant simultaneously monitors the changes occurring in the brain (i.e. changes in the chemical composition of cerebrospinal fluids etc). Similarly, the in-body implant records the changes happening in the target affected area at that particular moment. These implants communicate the changes the underlying biological layers undergo to the on-skin electrode through the process of "Galvanic coupling".

Galvanic coupling is a method for introducing an electrical communication signal to the body through a justified interface. The body plays the role of a communication channel and the injected administered signal is transmitted primarily through a skin medium [4]. Usually, the on-body devices interact wirelessly through radio frequency (RF) wave spectrum. However, galvanic coupling is a step further which provides a power efficient and a safer means of communication between the body implant and the on-surface electrodes. The communication involves two-way traffic:

\section{Surface-To-Implant Communication}

The signal transmitter of the communication system is placed on the skin surface and the signal receiver is embedded inside the biodegradable implant. The signal transmitter sends a current signal, and the signal reaches the receiving implantable device through the skin in order to achieve communication. The very method is utilized as a communication mode for electric energy transfer to the implanted device [3].

\section{Implant-To-Surface Communication}

In the implant-surface communication system, the current signal sent from the implanted device is current coupled with the on-body electrodes through the human tissue. Since the signal-source detecting device is executed in human body, the detected source signal is not affected by the surrounding environment [3].

In the above manner implants relay the gathered information to the on-skin electrodes periodically.

Now, since the on-skin electrodes and EEG headset are 'Time synced', the changes in the physiology of cells can be studied seamlessly at any instance of time by transferring the collected data (i.e. by the three separate components) wirelessly to the nearby mobile device that can analyze the corresponding data.

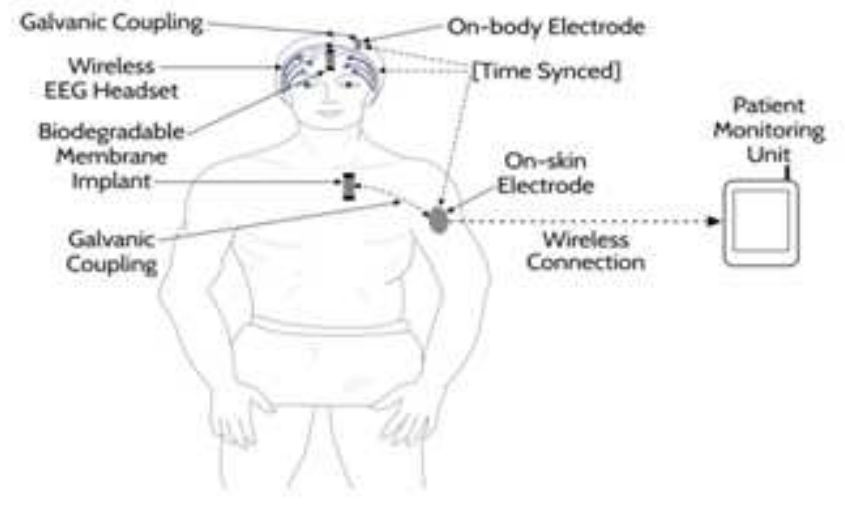

Figure 5: Learning the Impact of Positive/ Negative Emotions on Physiology of Human Cells

\section{PRELIMINARY RESEARCH FINDINGS}

Part: 1

It is identified in the recent research literature that the auditory BCI (Brain Computer Interface) is easier to learn 
the enhancement or lowering of the sensorimotor rhythm amplitude with visual feedback than with acoustic feedback alone. Another notable finding revealed that a typical healthy person with no eye problems usually have a less developed hearing sense than the one with visual defects. Conversely, it is also found that people with visual defects have an even better developed hearing sense than those with normal vision. Following this reasoning a combined effect of both visual and auditory stimuli are utilized to trigger an emotion for providing the best construct in emotion recognition.

Part: 2

Determining an optimal position for placement of a limited number of electrodes for emotion recognition Literature Survey.

Psycho-physiological research has identified the importance of the difference in activation of the two cortical hemispheres in response evoked by the test subjects towards the provided stimuli. Left frontal lobe inactivation is measure of a withdrawal response, which is considered as a negative thought process. On similar lines, right frontal lobe inactivation represents a positive thought process. High alpha activity $(8-12 \mathrm{~Hz}$ - as per EEG frequency records) exemplifies a manifestation of low brain activity, and vice versa. Thus, while considering cortical inactivation, in the EEG frequency band, an increase in alpha activity is observed, along with a fall in beta waves.

As per the observations made while scanning through the relevant literature - it was found that F3 and F4 are the most used electrode positions for looking at the above described alpha activity, as they are located above the 'dorsolateral prefrontal cortex'. Further, regarding the emotion in the brain, it is learnt that the 'prefrontal lobe' plays an influential role in emotion modulation and conscious experience. Furthermore, the brain activity in the subconscious mind is also monitored by tapping into the limbic system and the brain stem. In addition, the Vagus Nerve or Spindle neurons connecting the 'prefrontal cortex' to the Insula are also tracked since they are believed to be the communication superhighway that links the conscious to the subconscious as per the medical research study [12].

\section{CONCLUSIONS}

Thus by using the above invention we monitor the effects of human thoughts (+ve / -ve emotional states) on the target human cells. As the EEG electrodes and on-skin electrodes are 'time synched' - the effect of thoughts on the physiology of cells within the human body can be learnt in a detailed timely manner. So the mysterious "Placebo / Nocebo effect" can be examined by employing the novel technique.

\section{REFERENCES}

1. Bruce H. Lipton, Ph. D, "The Biology of Belief-Unleashing the Power of Consciousness, Matter \& Miracles", 2016.

2. "Bioresorbable silicon electronic sensors for the brain", Nature, University of Illinois at Urbana-Champaign, 4 FEBRUARY 2016 | VOL 530.

3. Shuang Zhang, et al, "Development and Prospect of Implantable Intra-body Communication Technology," Journal of Computers, Vol. 9, No. 2, February 2014

4. Ryan Gill, "Human Body Communication using Galvanic Coupling", ECE, 2017.

5. "MIT Implant Measures Tumor Growth", TechTalk - Serving the MIT community, MIT, Volume - 51, Number - 11, Wednesday - December 6, 2006.

6. Moseley, J. B., K. O' Malley, et al. (2002). “A Controlled Trial of Arthroscopic Surgery for Osteoarthritis of the Knee." New 
England Journal of Medicine 347(2): 81-88.

7. Discovery (2003). “Placebo: Mind Over Medicine?” Medical Mysteries. Silver Spring, MD, Discovery Health Channel.

8. Oschman, J. L. (2000).Chapter 9: Vibrational Medicine. "Energy Medicine: The Scientific Basis”. Edinburg, Harcourt Publishers: 121-137.

9. Priya R. Baghe, Blue Eyes Sensing Intelligences Technology Using Emotion Sensor, International Journal of Applied Engineering Research and Development (IJAERD), Volume 4, Issue 2, March - April 2014, pp. 113-122

10. Chu, S. (2002). “Cold atoms and quantum control.” Nature 416: 206-210.

11. Rumbles, G. (2001). “A laser turns down the heat.” Nature 409: 572-573.

12. “Reality - Based Brain-Computer Interaction”, Thesis for: Licentiate, Advisor: Lars-Erik Janlert (and Johan Eriksson), June 2011.

13. "Understanding the Conscious and the Subconscious Mind", Thomas C. Corley, January 1, 2016. 
